\title{
The Application of Cloud Computing in the Management of Digital Archives in Colleges and Universities
}

\author{
Yingle Yao \\ Zhengzhou University of Industrial Technology, Institute of Information Engineering, Xinzheng, \\ Zhengzhou, Henan Province, China, 451150 \\ 390169913@qq.com
}

Keywords: Cloud computing; Co-construction; Co-sharing; Archives resources integration; Digital archives of colleges and universities

\begin{abstract}
Based on the demand of integration and sharing of digital archives resources, this paper analyzes the fit and advantage of cloud computing and digital archives resource management, and puts forward a new idea of sharing and sharing digital archives resources in the cloud computing technology that make full use of cloud computing, virtualization, cloud storage technology to achieve a shared high, high security, good scalability, suitable for standardization of the shared file sharing database; Finally, through experimental analysis of traditional platforms and cloud computing platform performance Which proves that the use of cloud computing technology can effectively improve the university archives resources management related work.
\end{abstract}

\section{Introduction}

Cloud computing is the third change after PC, Internet information industry, but also a kind of business applications arising from the field of IT model. There are multiple versions of cloud definitions and concepts in different application areas and applications. National Institute of Standards and Technology released definition and explanation in September 2011 (National Institute of Standards and Technology of the cloud computing definition) on cloud computing are as follows: Cloud computing is a pay-per-use model, which allows users to easily and on-demand access to a shared, configurable set of computing resources (such as networks, servers, storage, applications, and services), takes management costs and minimal interaction with service providers to complete the rapid allocation and distribution of numerous computing resource collections. As a whole, the cloud model has five characteristics: self-service, seamless access, resource sharing, scalability and service quantification, and three service models: Software as a Service (SaaS) Platform as a Service (PaaS) and Infrastructure as Services (IaaS) [1].

\section{The Fit between Cloud Computing and University Archives Management}

Unified computing, unified storage, virtualization, security management is the key technology of cloud computing, the university archives also have similar characteristics of co-building and sharing, therefore use cloud computing technology to reform the archives of colleges and universities is also logical. Cloud computing-based digital archives use cloud computing technology to achieve service virtualization of university digital archives, which makes user task requests flexibly allocating to different resources, and optimizes the resource allocation according to the load condition, Users only need a lightweight client access to the cloud data center, you can get with the operating system and application-independent high-availability service environment.

The advantages of the service model of university digital archives based on cloud computing are mainly embodied in:(1) On-demand use, instant use of information services;(2) Fully mobilize the parallel computing power of the server cluster; (3) Open service interface: Users do not need to change the client environment; (4) High level of self-management; (5)To achieve dynamic adjustment of business processes. The cloud computing digital archives of colleges and universities run cooperatively and efficiently, and will make dynamic business processes according to the 
service contents and business logic of the archives to ensure the stability, maintainability and extensibility of archives service.

\section{Design of Management Framework of Cloud Computing Archives in Universities}

Constructing "Cloud Platform" Framework System of Archives Resources Construction in Colleges and Universities. Cloud computing technology has a positive impact on the change of the basic theory of archives, archives practice, The framework of digital archives resource in the cloud service environment is constructed, which includes system architecture, storage structure, security system, service platform and process management and guarantee mechanism. According to these contents, we design the corresponding calculation method to build the infrastructure system structure, system structure and calculation method, resource management security system, resource sharing service and application system of digital file resource sharing and management in cloud service platform [2]. The construction of these systems clustered digital archive resources in the cloud service architecture which distributed in the remote environment, calculated the sharing of resources metadata, packaged the existing archive resources in order to match system query, user needs for the digital archives resources "cloud service". Therefore, we shown the program in Fig. 1 based on the cloud service architecture.

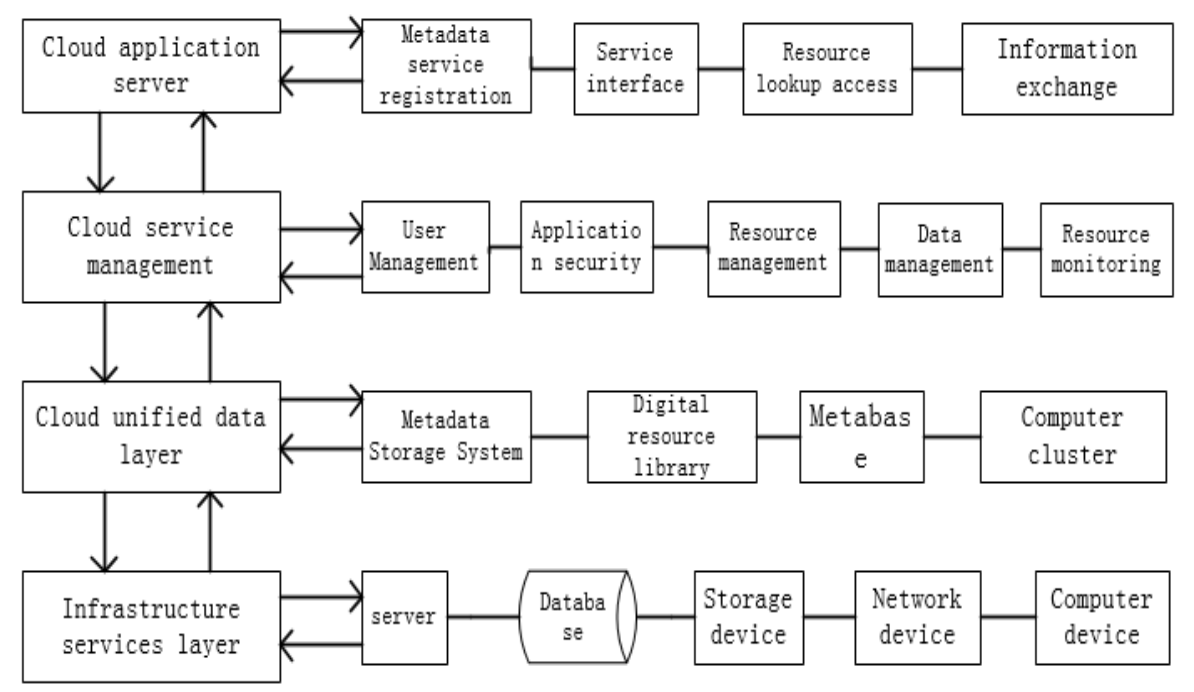

Figure 1. Cloud platform digital archives framework system

\section{Constructing "Cloud Storage" Platform for Jointly Building and Sharing University} Archives Resources. To build a unified cloud storage platform, we integrated the large number of university digital archives resources distributing in different regions and heterogeneous to management uniformly. Three points need to be considered: First, use virtualization technology, develop distributed digital archives data access interface base on "metadata" access, to achieve the conversion of heterogeneous digital archives resource data and "metadata". Based on the definition and storage structure of the existing digital archives resource data, a unified "metadata" access interface is provided for the upper layer call [3]. Second, to achieve dual-area off-site backup technology, divide into resources zone, each zone backup each other all the data, Once a regional failure can immediately go to another regional access or recovery, Data backup and disaster recovery can be realized without adding special backup equipment to improve the reliability of storage. Thirdly, it is necessary to design the concrete plan of the construction of "cloud storage" platform for digital archives resources sharing, to form a digital archives resource management platform which is open to the society, to build the metadata database and paper database resource sharing and management metadata database; developing transmission platform and encrypted tunnel among system and the digital file in order to achieve the digital file data encryption to protect the file information security, which is an important part of the research on digital file resource sharing 
and management system under cloud computing environment.

Constructing the "Cloud Service" Platform for Digital File Resource Sharing. To build a complete cloud service platform, four services are needed: access to digital archive resources, request authentication services, secure data transmission services, fast resource search and resource discovery services. First of all, to achieve access to digital archives resources need to develop a unified resource access interface to receive the application layer request, according to the parameters of the request, access the underlying cloud data, return the relevant "metadata" to the application layer; Second request authentication service is implemented: For a service request, according to the type of request, the authority of the application, in which the network environment, verify the legitimacy of the request. Again, secure data transfer services are implemented: For the data returned to the application, use 128-bit data encryption technology to encrypt the data, and then transmit; Finally, a fast resource search and resource discovery service is implemented: according to the parameters of the request, It can quickly search and find the resource area [4].Need to point out specially that the interface to provide services to the upper layer are used SOAP protocol and Web2.0 technology, compatible with a variety of terminals and operating systems.

Development of "Cloud Terminal" Application Software for Digital Archives Resource Construction. This paper analyzes the production process of digital archives resources and builds a metadata access and integration platform for digital archives resources by using the international metadata specification and standard (ISO/IEC11179), So as to integrate the heterogeneous digital archives resources to realize the unified management of the metadata of digital archives resources, and to ensure the effective transfer from the digital archives resources in the integration, retrieval and utilization. "Cloud Terminal" software is not aimed at an organization within, but for the whole social level, maximally realize the unity and coordination of various systems inside and outside the various aspects, so that all relevant agencies collaborate in large processes and standardize in a small process, restrict with a unified standard to play the overall advantages of digital archives resources, make integration, organization, archiving and a series of work in the approach of "cloud services" and also make retrieval and utilization of digital file. "Cloud Terminal" application software becomes the most cutting-edge management services window in the digital file sharing and management system.

\section{An Example of the Integration of University Archives Resources under the Cloud Platform}

Cloud Platform Digital Archives of Colleges and Universities Metadata Registration System Design. The primary goal of the Digital Archival Resource Metadata Registration System is to establish an authoritative, reliable, scalable and sustainable "cloud storage, cloud service" digital file resource metadata specification and its application system registration management mechanism [5]. In the "cloud services" platform, the functions of the digital archive resource meta-data registration system should include: standardizing the definition of metadata of archival resources, marking scheme, conversion rules, description rules, application guidelines, etc., and It supports the discovery, recognition, analysis, call, interface, search and exchange of digital archive resources metadata in cloud computing, and implements the metadata conversion, mining and integration of digital archives resources on the basis of this.

Design of "Cloud Service" Management System for Digital Archives in Colleges and Universities. Digital archives, the digital archives staff only need to deliver all the digital archives resources to the selected cloud service providers and sign the information security agreement with them to realize the digital archive resource information storage, No need to know the stored procedure, storage server location. However, because the archives resources are often involved in national security, information confidentiality and other issues, so all the digital archives resources information is not appropriate. The need for national archives in the work of planning a unified standard, classification of archival resources, identification, will not involve "confidentiality" of the file resources placed in the public "cloud computing", directly to users of digital archives resources retrieval, utilization and other services [6] . and will involve "confidentiality" is not easy to open the 
digital archives resource information, should be placed in the autonomy of private ownership of the "cloud", through the account login authentication can retrieve the use, so you can protect File resources, information security, based on the provision of intelligent, personalized file search services [7].

Design of Metadata Warehouse System for Digital Archives in Colleges and Universities. The International Organization for Standardization (ISO) metadata element specifications and standards (ISO / IEC 11179) support the sharing and reuse of data elements. The core problem of metadata management of digital archives resource is to establish a digital archive resource meta-data warehouse system, which can satisfy metadata access and life-cycle management system of digital archives resource. It is a platform for metadata access and integration of digital archives resources. And realize the integration of meta-data of remote digital archives resource. In addition, to establish a digital archive resource metadata exchange (digital archival resource metadata exchange protocol), digital file resource metadata between different systems can visit each other in this way, so that the heterogeneous digital Archives resources together to achieve unified management of digital archives metadata [8].

Design of University Digital Archives Resource Base. University teaching resource library is a unified data resource library, which needs to store teaching resources such as test paper library, courseware library, material library, etc. It also has a high requirement for data storage capacity, meanwhile, Some universities have a large amount of resources but a few high-quality resources, and some colleges and universities have a serious shortage of resources. How to make full use of the storage resources of colleges and universities to realize the sharing and sharing of teaching resources become a very urgent need to be solved. The paper puts forward the cloud storage of the teaching resource library, and makes use of the characteristics of the cloud storage to improve the storage capacity and processing capacity of the university data resource base. The storage structure is shown in

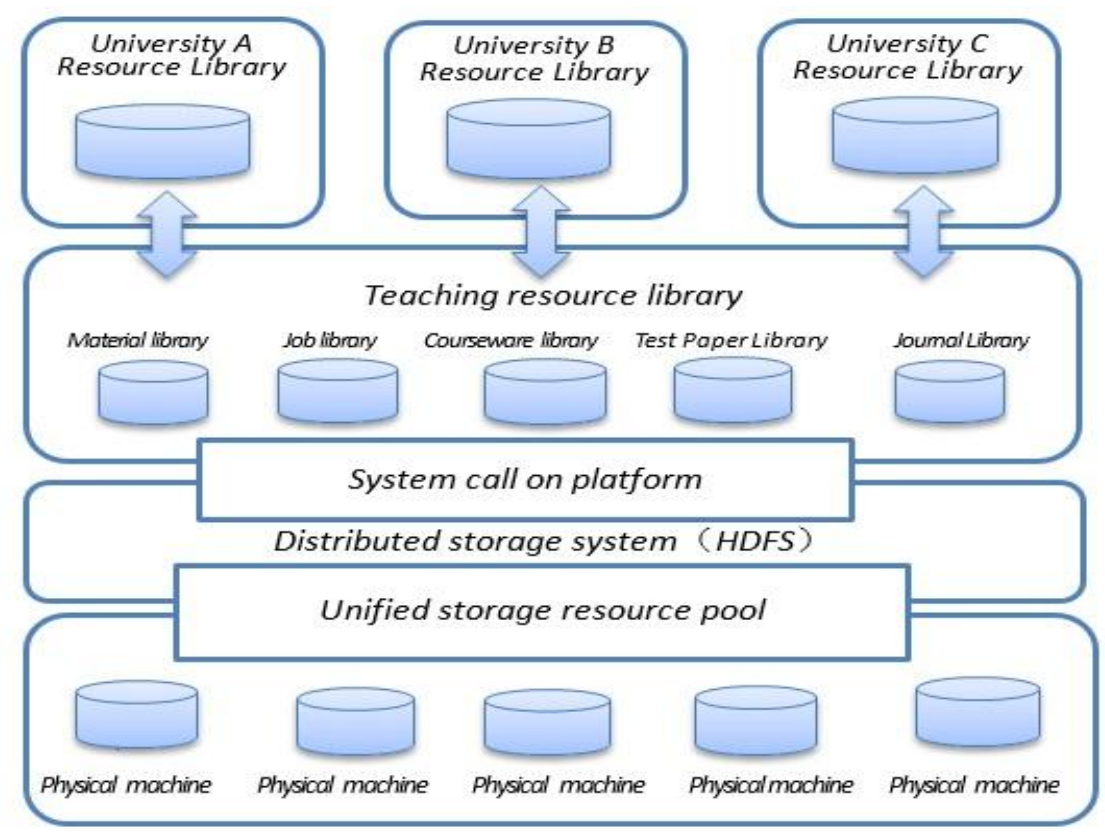

Fig. 2.

Figure 2. Storage structure of university digital archives resource base

Through the cloud storage technology to achieve the storage virtualization, to solve three problems [9]:

(1) To improve utilization. As a result of virtualization technology, according to the actual storage needs of various colleges and universities to dynamically allocate storage space, thus greatly improving the actual utilization of storage space.

(2) To improve sharing. By building a unified distributed teaching resource library, it is convenient to share the teaching resources in the resource pool, and also can upload the local data of 
the university resource library, enrich the construction of the teaching resource library, and highlight the sharing of teaching resources library construction. Sexual and co-construction.

(3) Improve security. Cloud storage has better data security, cloud storage provides a variety of data backup, can greatly improve the security of data storage.

\section{Experimental Environment Structures and Data Analysis}

In the light of the above related design ideas, through experiments to verify the traditional teaching resource library and cloud computing teaching resources library differences.

Experimental Environment Construction. The Hadoop cluster consists of one NameNode, one JobTraker and two DataNodes. The hardware configuration of all nodes is 1 ntel core $3.0 \mathrm{GHz}$, memory 4GB and hard disk 500GB. Table 1 shows the overall configuration.

Table 1 Hadoop server configuration table

\begin{tabular}{|c|c|c|c|}
\hline Serial number & IP address & Machine name & Node properties \\
\hline 1 & 192.168 .1 .100 & Hadoop1 & Master \\
\hline 2 & 192.168 .1 .101 & Hadoop2 & Slave \\
\hline 3 & 192.168 .1 .102 & Hadoop3 & Slave \\
\hline 4 & 192.168 .1 .103 & Hadoop4 & Slave \\
\hline
\end{tabular}

The Hadoop cluster adopts the master/slave mode. That is, the virtual compute nodes on the cloud platform include the master and the slave, where the master stores the metadata of the system and the slave node serves as the master node. As can be seen from the above table, the entire Hadoop cluster consists of a Master node and three Slave nodes. Through the Hadoop cluster system, you can build a basic cloud computing platform, any platform, users can request resources through the cloud platform [10].

Data Performance Analysis. The capacity of storage and retrieval of resources is a very important index in the construction of university archives resource base. The storage and retrieval capacity of massive archives resources under cloud platform are analyzed, the results are shown in Fig. 3 and Fig. 4.

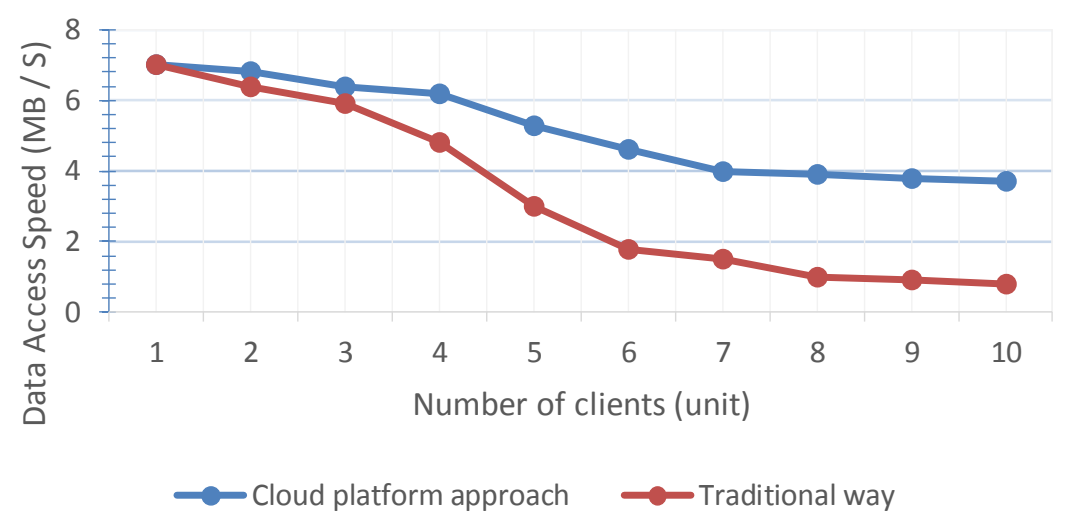

Figure 3. Analysis of storage capacity of archives 


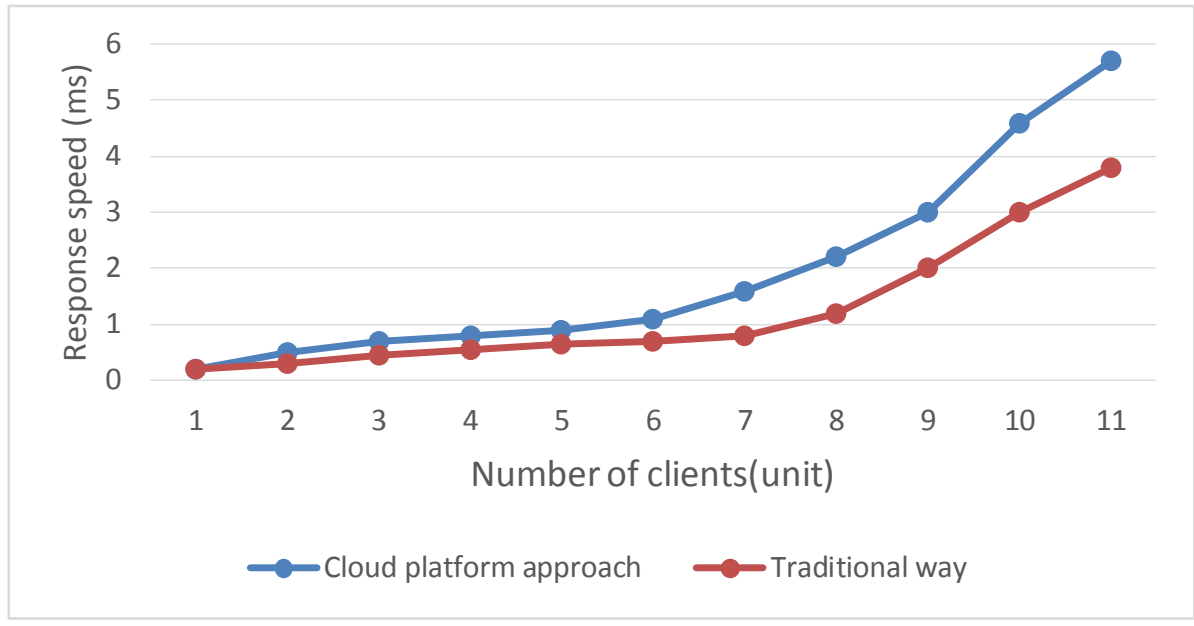

Figure 4. Analysis of retrieval capability of archival resource library

Can be seen, the traditional platform storage and cloud platform data storage differences. With the increasing number of users and data in traditional mode, the performance of data reading will decrease rapidly. Meanwhile, the response speed of the system drops seriously. The data access speed of the cloud platform is slower, and the response time to customers is also affected. Not much. In the cloud computing platform can also increase the number of virtual machines to improve data retrieval speed and system response time.

\section{Conclusion}

This paper deeply analyzes the application of cloud computing technology in the management of digital archives in universities. Experiments show that cloud computing technology can effectively improve the management of university archives resources:with the increasing in the number of clients, the storage capacity of archival resources, data retrieval speed, system response time is almost unaffected .But the shortcomings of cloud computing applications are less concerned, such as crash risk, data security and privacy, remains to be further studied.

\section{Acknowledgements}

First of all, I would like to thank the college leaders who gave me concern. Secondly, I would like to thank my colleague Zhang Yao, Zhang Kaiping, Mao Jianjing and Zhu Jiangbo for their encouragement and help in my thesis writing process; In this, please accept my sincere thanks. Finally, I want to thank my parents, my husband, my daughter; Thank you, my dear family.

\section{References}

[1] D.Y. Huang and X.P. Hu: Analysis and Application of Cloud Computing in Foreign Libraries [J]. Library and Information Service, Vol. 56 (2012) No.13, p.109. (In Chinese)

[2] R.Y. Deng, C. Qin and X.Z. Xie: Application of Mobile Cloud Computing and Analysis of Its Existing Problems [J]. Journal of Chongqing University of Posts and Telecommunications, Vol. 24 (2012) No.6, p.716. (In Chinese)

[3] W. Deng, F.M. Liu and H. Jin: New Energy Applications in Cloud Computing Data Center: Current Research and Trends [J] .Journal of Computer, Vol. 36 (2013) No.3, p.582. (In Chinese)

[4] H. Jin: Study on Characteristic Service of University Digital Library Based on Cloud Computing [J]. (2014) No.2, p.79. (In Chinese) 
[5] J. Wen: Study on the Construction of Digital Archives Based on Cloud Computing [J] .Journal of archives and construction, Vol. 5 (2011) No.1, p.46. (In Chinese)

[6] DinkarSitaram and GeethaManjunath. Migrating to the Cloud: Developing Applications in the New World of Cloud Computing [M] .Defense Industry Press, 2015.

[7] Z.P. Liu, N. Zhang and Z.L: Bian. Application of Cloud Computing Technology in Archives [J]. (2010) No.16, p.33. (In Chinese)

[8] J.J. Cheng: Study on Digital File Resource Sharing and Management System in Cloud Technology [J] .Journal of Archives, (2013) No.1, p.38. (In Chinese)

[9] Y.M. Yin: From Cloud Computing to Personal Digital Archives [J]. Shanxi Archives, (2009) No.2, p.23. (In Chinese)

[10] S.X. Xue and C. Huang: Review of Electronic Document Management in Cloud Computing Environment [J]. Beijing Archives, (2011) No.9, p.65. (In Chinese) 